standardised format created. Electronic forms were reviewed to ensure compliance with the updated documentation policy and a standardised abbreviation list created to ensure consistency.

Documentation training was introduced for new members of staff and a mandatory training programme provided using redacted patient records. Multi-disciplinary groups worked together to answer key clinical enquiries which were representative of issues investigated by a Coroner.

Results On-going audits demonstrate incremental improvement in documentation and to ensure standards are being maintained there are plans to introduce a peer documentation review process and record keeping champions.

Conclusion The development of a robust document management process, training and audit programme is fundamental to ensuring high standards of documentation and the delivery of high quality care.

\section{P-84 EXPLORATION OF ADULT HOSPICES AS A SHORT BREAK PROVIDER FOR YOUNG ADULTS WITH PALLIATIVE CARE NEEDS}

Helen Finlins. St Elizabeth Hospice, Ipswich, UK

10.1136/bmjspcare-2016-001245.107

\section{Background}

- In the UK the number of 16-25 year olds living with life limiting illness has doubled in the past decade (Fraser et al., 2011).

- Nationally there is a lack of suitable respite provision for young adults with palliative care needs (King and Barclay, 2007, p201).

- An integrative literature review has been carried out to support development of a business case for a regional young adult short break unit in an adult hospice.

- Short break provision is seen as integral to children's palliative care (Ling, 2012, p129) with many describing provision a "life line" (Jackson and Robinson, 2003, p105). Equivalent support does not exist in adult hospices.

- NICE Guidelines for Transition (2016) recommend developmentally appropriate care provision

Literature review-emerging themes Needs of parents:

- A break from complex and technical care is needed, to maintain well-being and enable time with other children

- A break enables parents to sustain care in the home

- Needs change over time-ageing parents and relatives, increasing complexity of care means less informal support available

- Parents struggle with trusting others to provide care.

Needs of young adults:

- Opportunities for valuable peer support

- Opportunity for social activities

- A break from family with opportunity to explore and increase independence from parents.

Significant paucity in primary research with young adults Service delivery
- Significant differences between children's and adult's hospice care-transition is challenging

- Young adult short break models have high care needs and expensive staffing costs

- Adult hospices lack experience in complex care needs of young adults-significant training needs.

Conclusions

- Significant respite needs for parents

- Developmentally appropriate respite should be available

- Children's hospices have often been the only provider able to meet these complex needs. Can adult hospices inherit this legacy for a new generation of young adults with palliative care needs surviving into adulthood?

- Further primary research with young adults needed.

\section{P-85 HALF THE COMMUNITY NURSE CASE LOAD!: ESTIMATING THE PREVALENCE OF LOWER LIMB CHRONIC OEDEMA}

${ }^{1}$ Margaret Benson, ${ }^{2}$ Rebecca Gaskin, ${ }^{2}$ Christine Moffatt, ${ }^{3}$ Victoria Peach, ${ }^{1}$ Christina Faull. ${ }^{1}$ LOROS Hospice, Leicester, UKi ${ }^{2}$ University of Nottingham; ${ }^{3}$ Leicester Partnership Trust

10.1136/bmjspcare-2016-001245.108

Background Chronic oedema has a profound impact on quality of life. It may originate from primary anatomical reasons (primary lymphoedema), be secondary to cardio-vascular dysfunction, be related to cancer or cancer treatments and is increasingly a result of obesity. The numbers of patients with chronic oedema are increasing and both hospice specialist lymphoedema and community nursing teams are over-stretched with 'revolving door referrals. The prevalence and burden of illness and impact on care services in the UK is unknown.

Aims

- To determine the scale and impact of lower limb chronic oedema and wounds within Leicester City.

- To inform the development of an integrated community chronic oedema pathway

Method Quantitative data was collected by community nurses following a period of training by the specialist hospice lymphoedema team. Each patient on a community nurse caseload was clinically assessed for chronic oedema and wounds and the results were recorded on a questionnaire.

Data validation was undertaken by a specialist lymphoedema nurse on a random sample of 20 patients establishing a high level of agreement between raters.

Results 1,308 patients were assessed. 43\% had chronic oedema defined by swelling and/or skin changes. $45 \%$ of these patients had an associated wound such as venous ulcer. Risk factor analysis is being undertaken comparing those with and without oedema.

Conclusion The prevalence of chronic oedema in the community nursing caseload is very high. The totality of the clinical care requirements for these patients were often unrecognised and dressings addressed as a 'task' in isolation of a clear management strategy. 